\title{
Information Theory vs. Queueing Theory for Resource Allocation in Multiple Access Channels
}

\author{
(Invited Paper) \\ Ali ParandehGheibi, Muriel Médard, Asuman Ozdaglar, and Atilla Eryilmaz
}

\begin{abstract}
We consider the problem of rate allocation in a fading Gaussian multiple-access channel with fixed transmission powers. The goal is to maximize a general concave utility function of the expected achieved rates of the users. There are different approaches to this problem in the literature. From an information theoretic point of view, rates are allocated only by using the channel state information. The queueing theory approach utilizes the global queue-length information for rate allocation to guarantee throughput optimality as well as maximizing a utility function of the rates. In this work, we make a connection between these two approaches by showing that the information theoretic capacity region of a multiple-access channel and its stability region are equivalent. Moreover, our numerical results show that a simple greedy policy which does not use the queue-length information can outperform queue-length based policies in terms of convergence rate and fairness.
\end{abstract}

\section{INTRODUCTION}

Dynamic allocation of communication resources such as bandwidth or transmission power is a central issue in multiple access channels (MAC) in view of the time varying nature of the channel and interference effects. Most of the existing literature on resource allocation in multiple access channels focuses on specific communication schemes such as TDMA (time-division multiple access) [1] and CDMA (code-division multiple access) [2], [3] systems. An exception is the work by Tse et al. [4], who introduced the notion of throughput capacity for the fading channel with Channel State Information (CSI) and studied dynamic rate allocation policies with the goal of maximizing a linear utility function of rates over the throughput capacity region.

Another important literature relevant to our work appears in the context of cross-layer design, where joint schedulingrouting-flow control algorithms have been proposed and shown to achieve utility maximization for concave utility functions while guaranteeing network stability (e.g. [5], [6], [7], [8]).

This research was partially supported by the National Science Foundation under grant DMI-0545910, and by DARPA ITMANET program.

A. ParandehGheibi is with the Laboratory for Information and Decision Systems, Electrical Engineering and Computer Science Department, Massachusetts Institute of Technology, Cambridge MA, 02139 (e-mail: parandeh@mit.edu)

A. Ozdaglar and M. Médard are with the Laboratory for Information and Decision Systems, Electrical Engineering and Computer Science Department, Massachusetts Institute of Technology, Cambridge MA, 02139 (e-mails: asuman@mit.edu, medard@mit.edu)

A. Eryilmaz is with the Electrical and Computer Engineering, Ohio State University, OH, 43210 (e-mail: eryilmaz@ece.osu.edu)
The common idea behind these schemes is to use properly maintained queues to make dynamic decisions about new packet transmission as well as rate allocation.

Some of these works ([6], [7]) explicitly address the fading channel conditions, and show that the associated policies can achieve rates arbitrarily close to the optimal based on a design parameter choice. Requiring global queue-length information may impose much more overhead on the system than channel state information, because for a slow fading channel the sampling rate for channel estimation is significantly smaller than the data arrival rate which dictates the necessary sampling rate for obtaining the queue-length information.

In this paper, we consider the problem of rate allocation in a multiple access channel with perfect CSI. We assume that the transmitters do not have power control feature, and no prior knowledge of channel statistics is available. We first relates the information theory and queueing theory approaches by showing the equivalence between the information theoretic capacity region of a multiple-access channel and its stability region. Hence, we conclude that the policies assigning rates form the capacity region of a multiple-access channel are indeed throughput optimal.

We consider the utility maximization framework with a general concave utility function of the rates to model different performance metrics and fairness criteria (cf. Shenker [9], Srikant [10]). We present an efficient greedy rate allocation policy [11] which is in general suboptimal but does not require queue-length information, and compare its performance with the queue-length based policy by Eryilmaz and Srikant [6] for different scenarios with limited communication duration.

The remainder of this paper is organized as follows: In Section II, we introduce the model and describe the capacity region of a multiple-access channel. In Section III, we relate the information theory and queueing theory approaches to resource allocation. In Section IV, we present a dynamic rate allocation policy designed by each of theses approaches. In Section V, we provide the simulation results to compare the performance of the presented policies under different scenarios. Finally, we give our concluding remarks in Section VI.

\section{SYSTEM MODEL}

We consider $M$ users sharing the same media to communicate to a single receiver. We model the channel as a Gaussian 
multiple access channel with flat fading effects

$$
Y(t)=\sum_{i=1}^{M} \sqrt{H_{i}(t)} X_{i}(t)+Z(t),
$$

where $X_{i}(t)$ are the transmitted waveform with average power $P_{i}, H_{i}(t)$ is the channel gain corresponding to the $i$-th user and $Z(t)$ is white Gaussian noise with variance $N_{0}$. We assume that the channel gains are known to all users and the receiver 1. Throughout this work we assume that the transmission powers are fixed and no prior knowledge of channel statistics is available.

First, consider the non-fading case where the channel gains are fixed. The capacity region of the Gaussian multiple-access channel with no power control is described as follows [12]

$$
\begin{array}{r}
C_{g}(\boldsymbol{P}, \boldsymbol{h})=\left\{\boldsymbol{R} \in \mathbb{R}_{+}^{M}: \sum_{i \in S} R_{i} \leq C\left(\sum_{i \in S} h_{i} P_{i}, N_{0}\right),\right. \\
\text { for all } S \subseteq \mathcal{M}=\{1, \ldots, M\}\},
\end{array}
$$

where $P_{i}$ and $R_{i}$ are the $i$-th transmitter's power and rate, respectively. $C(P, N)$ denotes Shannon's formula for the capacity of an AWGN channel given by

$$
C(P, N)=\frac{1}{2} \log \left(1+\frac{P}{N}\right) \text { nats. }
$$

For a multiple-access channel with fading, but fixed transmission powers $P_{i}$, the throughput capacity region is given by averaging the instantaneous capacity regions with respect to the fading process [13],

$$
\begin{array}{r}
C_{a}(\boldsymbol{P})=\left\{\boldsymbol{R} \in \mathbb{R}_{+}^{M}: \sum_{i \in S} R_{i} \leq \mathbb{E}_{\boldsymbol{H}}\left[C\left(\sum_{i \in S} H_{i} P_{i}, N_{0}\right)\right],\right. \\
\text { for all } S \subseteq\{1, \ldots, M\}\},
\end{array}
$$

where $\mathbb{E}_{\boldsymbol{H}}$ denotes the expectation with respect to $\boldsymbol{H}$, that is a random vector with the stationary distribution of the fading process. Under the utility maximization framework, the goal is to find optimal rate allocation policy with respect to the utility function, $u(\cdot)$, as defined in the following.

Definition 1: [Optimal Policy] The optimal rate allocation policy denoted by $\mathcal{R}^{*}: \mathbb{R}^{M} \rightarrow \mathbb{R}^{M}$ is a mapping that satisfies $\mathcal{R}^{*}(\boldsymbol{H}) \in C_{g}(\boldsymbol{P}, \boldsymbol{H})$ for all $\boldsymbol{H}$, and

$$
\mathbb{E}_{\boldsymbol{H}}\left[\mathcal{R}^{*}(\boldsymbol{H})\right]=\boldsymbol{R}^{*} \in \quad \begin{array}{ll}
\operatorname{argmax} & u(\boldsymbol{R}) \\
\text { subject to } & \boldsymbol{R} \in C_{a}(\boldsymbol{P}) .
\end{array}
$$

\section{StABILITy REgion AND INFORMATION THEORETIC CAPACITY REGION}

In this part, first we make a connection between information theory and queueing theory approaches by showing the information theoretic capacity region of a multiple access channel and its stability region coincide. The stability region

\footnotetext{
${ }^{1}$ This is assumption is satisfied in practice when the receiver measures the channels and feeds back the channel information to the users.
}

is defined as the closure of the set of arrival rates for which there is a service scheduling policy stabilizing the queues, i.e., $\lim \sup _{t \rightarrow \infty} \mathbb{E}\left[Q_{i}(t)\right]<\infty$, where $Q_{i}(t)$ is the queue length of user $i$. This approach is similar to the work of Médard $e t$ al. [14] in which stability of a time-slotted Aloha system is established, regardless of the burstiness of the traffic. Let us first introduce a useful lemma known as T-slot Lyapunov drift that is widely used in stability analysis of queueing systems.

Lemma 1: (T-slot Lyapunov drift $[15])$ Let $\boldsymbol{Q}(t) \in \mathbb{R}^{M}$ denote the queue-lengths in a network with $M$ users. If there exists a positive integer $T$ such that $\mathbb{E}[\boldsymbol{Q}(\tau)]<\infty$ for $\tau \in$ $\{1, \ldots, T-1\}$, and if there are positive scalars $\epsilon$ and $B$ such that for all time slots $t_{0}$ we have:

$$
\mathbb{E}\left[V\left(\boldsymbol{Q}\left(t_{0}+T\right)\right)-V\left(\boldsymbol{Q}\left(t_{0}\right)\right) \mid \boldsymbol{Q}\left(t_{0}\right)\right] \leq B-\epsilon \sum_{i=1}^{M} Q_{i}\left(t_{0}\right),
$$

for some Lyapunov function $V: \mathbb{R}^{M} \rightarrow \mathbb{R}$, then the network is strongly stable, i.e., the queue-lengths satisfy:

$$
\limsup _{t \rightarrow \infty} \frac{1}{t} \sum_{\tau=0}^{t-1} \sum_{i=1}^{M}\left[Q_{i}(\tau)\right] \leq \frac{B}{\epsilon}
$$

Next, we present a new result which establishes the equivalence between the capacity region and stability region of a Gaussian MAC.

Theorem 1: Consider a $M$-user Gaussian multiple access channel. Let $A_{i}(t)$ be the arrival rate of the $i$-th user at time slot $t$, where $\mathbb{E}\left[A_{i}(t)\right]=\lambda_{i}$ and $\mathbb{E}\left[A_{i}^{2}(t)\right]<\infty$. Then the capacity region of the channel coincides with its stability region.

Proof: Let $\Lambda$ and $C$ denote the stability region and information theoretic capacity region of the channel, respectively. First, we show that $C \subset \Lambda$ by presenting a simple achievable scheme based on achievability proof of MAC capacity region which guarantees stability of the queues for any achievable rate tuple.

Assume for the expected arrival rate $\boldsymbol{\lambda}$ we have $\boldsymbol{\lambda} \in \operatorname{int}(C)$. Then, there exists a rate tuple $\boldsymbol{R} \in \operatorname{int}(C)$ such that $R_{i}=$ $\lambda_{i}+\epsilon$, for some $\epsilon>0$. Since $\boldsymbol{R}$ lies in the interior of $C$, it is achievable, i.e., for every $P_{e}>0$ there exists large enough $N$ such that user $i$ can transmit $N R_{i}$ bits of information by $N$ channel use with probability of error smaller than $P_{e}$. For the given rate tuple $\boldsymbol{R} \in \operatorname{int}(C)$, choose $n$ large enough such that

$$
P_{e} \leq \frac{\epsilon}{2\left(\max _{i} \lambda_{i}+\epsilon\right)} .
$$

Using the Foster's criterion for stability, we show the following scheduling algorithm stabilizes the queues. User $i$ starts transmitting only if $Q_{i}(t)-n R_{i}<0$, where $Q_{i}(t)$ denotes the queue-length of user $i$ at time $t$. In this case user $i$ chooses a codeword from its codebook of size $2^{n R_{i}}$ and transmits it over the channel by $n$ channel use.

Note that in this scheme users transmit asynchronously. However, the information theoretic capacity region remains the same [16]. The $i$-th user's queue dynamics are given by

$$
Q_{i}(t+n)=\left\{\begin{array}{lr}
Q_{i}(t)+\sum_{\tau=t}^{t+n-1} A_{i}(\tau), & Q_{i}(t)<n R_{i} \\
Q_{i}(t)-n R_{i} S_{i}(t)+\sum_{\tau=t}^{t+n-1} A_{i}(\tau), & \text { otherwise. }
\end{array}\right.
$$


where $Q_{i}(t)$ denotes the queue-length of user $i$, and $S_{i}(t)$ are Bernoulli random variables that take value 0 with probability $P_{e}$. Let $V(\boldsymbol{Q})=\sum_{i=1}^{M} Q_{i}^{2}$ be the Lyapunov function, and define the $n$-slot drift as follows:

$$
\begin{aligned}
\Delta_{n}(\boldsymbol{Q}(t)) & =\mathbb{E}[V(\boldsymbol{Q}(t+n))-V(\boldsymbol{Q}(t)) \mid \boldsymbol{Q}(t)] \\
& =\sum_{i=1}^{M} \mathbb{E}\left[Q_{i}^{2}(t+n)-Q_{i}^{2}(t) \mid \boldsymbol{Q}(t)\right] .
\end{aligned}
$$

Given the queue dynamics in (7), we can bound each term in (9) as follows. For the case that $Q_{i}(t) \geq n R_{i}=n\left(\lambda_{i}+\epsilon\right)$, we have

$$
\begin{aligned}
\mathbb{E}[ & \left.Q_{i}^{2}(t+n)-Q_{i}^{2}(t) \mid \boldsymbol{Q}(t)\right] \\
= & \mathbb{E}\left[\left(Q_{i}(t+n)-Q_{i}(t)\right)\left(Q_{i}(t+n)+Q_{i}(t)\right) \mid \boldsymbol{Q}(t)\right] \\
= & \mathbb{E}\left[2 Q_{i}(t)\left(\sum_{\tau=t}^{t+n-1} A_{i}(\tau)-n R_{i} S_{i}(t)\right) \mid \boldsymbol{Q}(t)\right] \\
& +\mathbb{E}\left[\left(\sum_{\tau=t}^{t+n-1} A_{i}(\tau)-n R_{i} S_{i}(t)\right)^{2} \mid \boldsymbol{Q}(t)\right] \\
= & 2 Q_{i}(t)\left(n \lambda_{i}-n\left(\lambda_{i}+\epsilon\right)\left(1-P_{e}\right)\right) \\
& +n E\left[A_{i}^{2}(t)\right]+n(n-1) \lambda_{i}^{2}+n^{2} R_{i}^{2}\left(1-P_{e}\right) \\
& -2 n^{2} \lambda_{i} R_{i}\left(1-P_{e}\right) \\
= & 2 n Q_{i}(t)\left(P_{e}\left(\lambda_{i}+\epsilon\right)-\epsilon\right)+B_{i}^{\prime} \\
\leq & -(n \epsilon) Q_{i}(t)+B_{i}^{\prime}=-\epsilon^{\prime} Q_{i}(t)+B_{i}^{\prime},
\end{aligned}
$$

where $B_{i}^{\prime} \leq n \mathbb{E}\left[A_{i}^{2}(t)\right]+n^{2}\left(P_{e} \lambda_{i}+\epsilon R_{i}\right)<\infty$, and the inequality follows from (6).

Now consider the case that $Q_{i}(t)<n R_{i}$, where $n$ is a fixed finite number. Since the queue-length is bounded at time $t$, it will remain bounded at time $t+n$, even if no successful transmission occurs between $t$ and $t+n$. Thus, we can write a negative drift for $Q_{i}$. In particular

$$
\begin{aligned}
\mathbb{E} & {\left[Q_{i}^{2}(t+n)-Q_{i}^{2}(t) \mid \boldsymbol{Q}(t)\right] } \\
& =\mathbb{E}\left[2 Q_{i}(t)\left(\sum_{\tau=t}^{t+n-1} A_{i}(\tau)\right) \mid \boldsymbol{Q}\right]+\mathbb{E}\left[\left(\sum_{\tau=t}^{t+n-1} A_{i}(\tau)\right)^{2} \mid \boldsymbol{Q}\right] \\
& =2 Q_{i}(t)\left(n \lambda_{i}-\frac{n \epsilon}{2}+\frac{n \epsilon}{2}\right)+n \mathbb{E}\left[A_{i}^{2}(t)\right]+n(n-1) \lambda_{i}^{2} \\
& =-(n \epsilon) Q_{i}(t)+n^{2} R_{i}\left(2 \lambda_{i}+\epsilon\right)+n \mathbb{E}\left[A_{i}^{2}(t)\right]+n(n-1) \lambda_{i}^{2} \\
& \leq-\epsilon^{\prime} Q_{i}(t)+B_{i}^{\prime \prime},
\end{aligned}
$$

where $B_{i}^{\prime \prime}=n^{2} R_{i}\left(2 \lambda_{i}+\epsilon\right)+n \mathbb{E}\left[A_{i}^{2}(t)\right]+n(n-1) \lambda_{i}^{2}<\infty$.

The total Lyapunov drift can be written as

$$
\begin{aligned}
\Delta_{n}(\boldsymbol{Q}(t)) & =\sum_{i=1}^{M} \mathbb{E}\left[Q_{i}^{2}(t+n)-Q_{i}^{2}(t) \mid \boldsymbol{Q}(t)\right] \\
& \leq-\epsilon^{\prime} \sum_{i=1}^{M} Q_{i}(t)+B
\end{aligned}
$$

where $B=\sum_{i=1}^{M} \max \left\{B_{i}^{\prime}, B_{i}^{\prime \prime}\right\}<\infty$.
Therefore, by Lemma 1, the network is stable for any arrival rate $\boldsymbol{\lambda}$ in the interior of the information theoretic capacity region. Hence, $C \subset \Gamma$.

For the converse part, suppose $\lambda \notin C$. We show that no scheduling policy can stabilize the queues. Since $\lambda \notin C$, there exists some $S \subseteq \mathcal{M}$ such that

$$
\sum_{i \in S} \lambda_{i} \geq C\left(\sum_{i \in S} P_{i}, N_{0}\right)+\epsilon,
$$

for some $\epsilon>0$. It follows from Fano's inequality [12] that transmission rate tuple $\boldsymbol{R}$ per channel use and the probability of error, $P_{e}$, satisfy the following

$$
\sum_{i \in S} R_{i} \leq C\left(\sum_{i \in S} P_{i}, N_{0}\right)+P_{e} \sum_{i \in S} R_{i}
$$

Therefore, by the Strong Law of Large Numbers we have

$$
\begin{aligned}
\sum_{i \in S} Q_{i}(t) & =\sum_{i \in S} \sum_{\tau=0}^{t-1} A_{i}(\tau)-t \sum_{i \in S} R_{i}\left(1-P_{e}\right) \\
& =t \sum_{i \in S} \lambda_{i}-t\left(1-P_{e}\right) \sum_{i \in S} R_{i} \\
& \geq t\left(C\left(\sum_{i \in S} P_{i}, N_{0}\right)+\epsilon\right)-t C\left(\sum_{i \in S} P_{i}, N_{0}\right)=t \epsilon,
\end{aligned}
$$

where the inequality directly follows from (11). By taking the limit as $t$ goes to infinity, we conclude that not all the queues in the network can be stabilized. This establishes the converse and hence, equivalence of the stability region and information theoretic region of the multiple access channel.

The proof of Theorem 1 simply generalizes for the case of fading multiple access channel using the achievability proof of fading MAC and by taking the expectations with respect to the fading process. Next, we present dynamic rate allocation policies designed from information theory and queueing theory point of view.

\section{Dynamic Rate Allocation Policies}

In this section, we first present a greedy rate allocation policy that only uses the instantaneous channel state information to allocate rates to different users.

Definition 2: [Greedy Policy] A greedy rate allocation policy, denoted by $\overline{\mathcal{R}}$, is given by

$$
\overline{\mathcal{R}}(\boldsymbol{H})=\begin{array}{cl}
\operatorname{argmax} & u(\boldsymbol{R}) \\
\text { subject to } & \boldsymbol{R} \in C_{g}(\boldsymbol{P}, \boldsymbol{H})
\end{array}
$$

i.e., for each channel state, the greedy policy chooses the rate vector that maximizes the utility function over the corresponding capacity region.

This policy can be implemented efficiently by exploiting polymatroid structure of MAC capacity region leading to a gradient projection method with approximate projection [17]. Note that the greedy policy is not necessarily optimal for general concave utility functions. However, it can be shown [11] that under some mild technical assumptions, the performance difference between the greedy and the optimal policy (cf. Definition 1) 


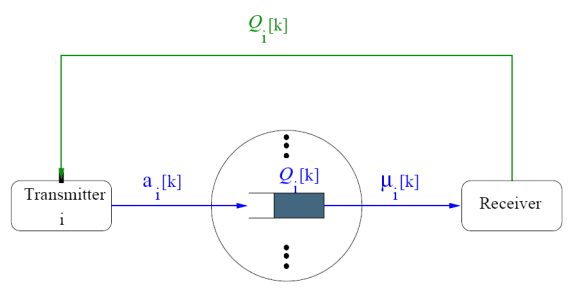

Fig. 1. Structure of the $i$-th transmitter and the receiver for the queue-lengthbased policy [6].

is bounded. This bound goes to zero as the utility tends to a linear function or the channel variations vanish.

Now, we present a rate allocation policy for fading multiple access channel by Eryilmaz and Srikant [6]. This policy explicitly uses queue-length information in order to guarantee stability of the network and also captures some fairness issues by considering the following utility function:

$$
u(\boldsymbol{R})=\sum_{i} w_{i} f_{\alpha}\left(R_{i}\right)
$$

where $f_{\alpha}(\cdot)$ denotes an $\alpha$-fair function given by

$$
f_{\alpha}(x)= \begin{cases}\frac{x^{1-\alpha}}{1-\alpha}, & \alpha \neq 1 \\ \log (x), & \alpha=1\end{cases}
$$

As illustrated in Figure 1, $Q_{i}(t)$ is the queue-length of the $i$-th user. The evolution of the $i$-th queue is given by

$$
Q_{i}(t+1)=\left(Q_{i}(t)+a_{i}(t)-\mu_{i}(t)\right)^{+}
$$

where $a_{i}(t)$ and $\mu_{i}(t)$ denote the arrivals and service of queue $i$, respectively. At time slot $t$, the scheduler chooses the service rate vector $\boldsymbol{\mu}(t)$ based on a max-weight policy, i.e.,

$$
\begin{aligned}
& \boldsymbol{\mu}(t)=\quad \operatorname{argmax} \sum_{i=1}^{M} Q_{i}(t) R_{i} \\
& \text { subject to } \quad \boldsymbol{R} \in C_{g}(\boldsymbol{P}, \boldsymbol{H}(t)) \text {. }
\end{aligned}
$$

The congestion controller proposed in [6] leads to a fair allocation of the rates for a given $\alpha$-fair utility function. In particular, the data generation rate for the $i$-th transmitter, denoted by $a_{i}(t)$ is a random variable satisfying the following conditions:

$$
\begin{aligned}
& \mathbb{E}\left[a_{i}(t) \mid Q_{i}(t)\right]=\min \left\{K\left(\frac{w_{i}}{Q_{i}(t)}\right)^{\frac{1}{\alpha}}, D\right\}, \\
& \mathbb{E}\left[a_{i}^{2}(t) \mid Q_{i}(t)\right] \leq U<\infty, \text { for all } Q_{i}(t),
\end{aligned}
$$

where $\alpha, D$ and $U$ are positive constants. The following theorem from [6] states that the parametric rate allocation policy presented above is asymptotically optimal as $K$ grows to infinity.

Theorem 2: Let $\boldsymbol{R}^{*}$ be the optimal solution of the optimization problem (5). The mean stationary service rate vector converges to $\boldsymbol{R}^{*}$ as $K$ increases, i.e.,

$$
\left|\bar{\mu}_{i}-R_{i}^{*}\right| \leq B(K)^{-\alpha / 2}
$$
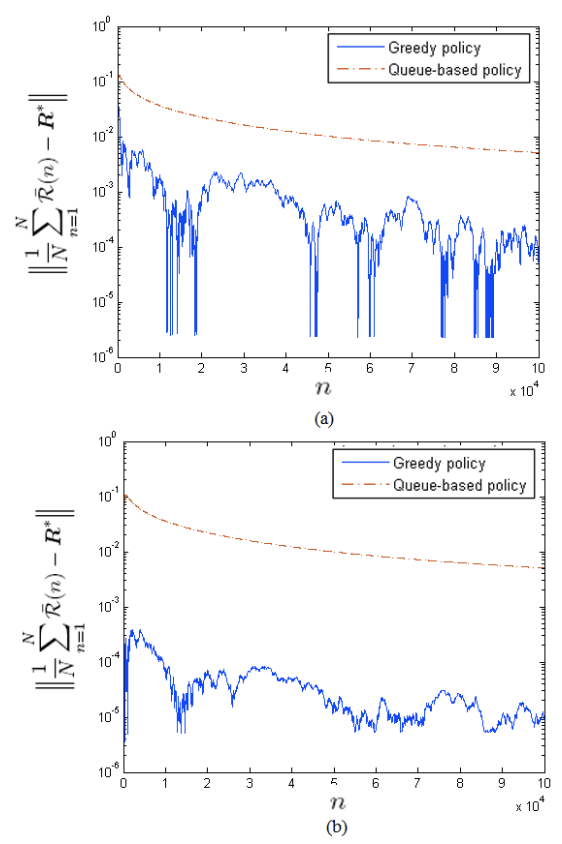

Fig. 2. Performance comparison of the greedy and the queue-based policies for a communication session with limited duration, for (a) $\frac{\sigma_{H}}{\bar{H}}=1.22$ (b) $\frac{\sigma_{H}}{H}=0.13$.

where $B$ is a finite positive constant and $\bar{\mu}$ is the mean service rate provided by the rate allocation algorithm under the stationary distribution of the Markov chain. Moreover, for any initial queue-length vector $\boldsymbol{Q}(0)$, we have

$\lim _{T \rightarrow \infty} \frac{1}{T} \sum_{t=0}^{T-1} \min \left\{K\left(\frac{w_{i}}{Q_{i}(t)}\right)^{\frac{1}{\alpha}}, D\right\}=\overline{\boldsymbol{\mu}}, \quad$ almost surely.

The proof and further details are given in [6]. In the following, we shall study the performance of this policy further using numerical results in the following section.

\section{Simulation Results and Discussion}

In this section, we provide simulation results to compare performance of the rate allocation policies presented in Section IV. For this simulations, we make the reasonable assumption that the channel state processes are generated by independent identical finite state Markov chains.

We consider two different scenarios to compare the performance of the greedy policy with the queue-based rate allocation policy by Eryilmaz and Srikant [6]. This policy, parameterized by some parameter $K$, uses queue length information to allocated the rates arbitrarily close to the optimal policy by choosing $K$ large enough. The greedy policy as defined in Definition 2 only uses the channel state information and does not require queue-length information for dynamic rate allocation.

In the first scenario, we compare the average achieved rate of the policies for a communication session with limited duration. Figure 2(a) depicts the distance between empirical average achieved rate of greedy or queue-length based policy and $\boldsymbol{R}^{*}$, 


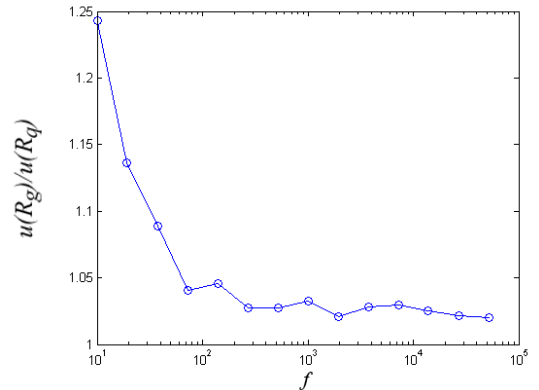

Fig. 3. Performance comparison of greedy and queue-based policies for file upload scenario with respect to file size $f=f_{1}=f_{2} . R_{g}$ and $R_{q}$ are expected upload rate of the greedy and the queue-length based policy, respectively.

the maximizer of the utility function over throughput region. In this case, the utility function is given by (13) with $\alpha=2$ and $w_{1}=1.5 w_{2}=1.5$, and the corresponding optimal solution is $\boldsymbol{R}^{*}=(0.60,0.49)$. As observed in Figure 2(a), the greedy policy outperforms the queue-length based policy for limited duration of the communication session. It is worth noting that there is a tradeoff in choosing the parameter $K$ of the queuelength based policy. In order to guarantee achieving close to optimal rates by queue-based policy, the parameter $K$ should be chosen large which results in large expected queue length and lower convergence rate. On the other hand, if $K$ takes a small value to improve the convergence rate, the expected achieved rate of the queue based policy lies in a larger neighborhood of the $\boldsymbol{R}^{*}$.

Furthermore, the performance of the greedy policy improves by decreasing the channel variations. Figure 2(b) demonstrates the improvement in performance of the greedy policy when the total channel variations, $\frac{\sigma_{H}}{H}$, has decreased from 1.22 to 0.13 . We also observe in Figure 2(b) that the queue-length based policy does not improve by decreasing channel variations.

Second, we consider a file upload scenario where each user transmits a file with finite size to the base station in a rateless manner. Let $\mathcal{T}_{i}$ be the $i$-th user's completion time of the file upload session for a file of size $f_{i}$. Define the average upload rate for the $i$-th user as $\frac{f_{i}}{\mathcal{T}_{i}}$. We can measure the performance of each policy by evaluating the utility function at the average upload rate. Figure 3 illustrates the utility difference of the greedy and the queue-based policy for different file sizes. We observe that for small file sizes the greedy policy outperforms the queue-based policy significantly, and this difference decrease by increasing the file size. We can interpret this behavior as follows. The files are first buffered into the queues based on the queue lengths and the weighted $\alpha$-fair utility, while the queues are emptied by a max-weight scheduler. Once the files are all buffered in the queues, the queues empty with the same rate which is not fair because it does not give any priority to the users based on their utility. For larger file sizes, the duration for which the queue is non-empty but the file is totally buffered in the queue, is negligible compared to total transmission time, and the average upload rate converges to a nearly optimal rate.

\section{CONCLUSION}

We addressed the problem of rate allocation in a fading multiple access channel with no power control and prior knowledge of channel statistics, and considered the utility maximization framework for a general concave utility function of the rates. We made a connection between the information theory and queueing theory approaches to this problem by showing the equivalence relation between the capacity region of a multiple access channel and its stability region.

We also presented dynamic rate allocation policies designed by each approach and compared their performance using the simulation results for a communication scenario with limited duration or limited file size. The numerical results show that a simple greedy policy that does not use the queue-length information can outperform the queue-length based policies in terms of convergence rate and fairness.

\section{REFERENCES}

[1] X. Wang and G.B. Giannakis. Energy-efficient resource allocation in time division multiple-access over fading channels. Preprint, 2005.

[2] S.J. Oh, Z. Danlu, and K.M. Wasserman. Optimal resource allocation in multiservice CDMA networks. IEEE Transactions on Wireless Communications, 2(4):811-821, 2003.

[3] J.B. Kim and M.L. Honig. Resource allocation for multiple classes of DSCDMA traffic. IEEE Transactions on Vehicular Technology, 49(2):506519, 2000.

[4] D. Tse and S. Hanly. Multiaccess fading channels part I: Polymatroid structure, optimal resource allocation and throughput capacities. IEEE Transactions on Information Theory, 44(7):2796-2815, 1998.

[5] X. Lin and N. Shroff. The impact of imperfect scheduling on crosslayer rate control in multihop wireless networks. In Proceedings of IEEE Infocom, Miami, FL, March 2005.

[6] A. Eryilmaz and R. Srikant. Fair resource allocation in wireless networks using queue-length based scheduling and congestion control. In Proceedings of IEEE Infocom, volume 3, pages 1794-1803, Miami, FL, March 2005.

[7] M.J. Neely, E. Modiano, and C. Li. Fairness and optimal stochastic control for heterogeneous networks. In Proceedings of IEEE Infocom, pages 1723-1734, Miami, FL, March 2005.

[8] A. Stolyar. Maximizing queueing network utility subject to stability: Greedy primal-dual algorithm. Queueing Systems, 50(4):401-457, 2005.

[9] S. Shenker. Fundamental design issues for the future internet. IEEE Journal on Selected Areas in Communications, 13:1176-118, 1995.

[10] R. Srikant. Mathematics of Internet Congestion Control. Birkhauser, 2004.

[11] A. ParandehGheibi, A. Eryilmaz, A. Ozdaglar, and M. Médard. Rate and power allocation in fading multiple access channels. Proceedings of WiOpt, 2008.

[12] T.M. Cover and J.A. Thomas. Elements of Information Theory. John Wiley and Sons, Inc., New York, New York, 1991.

[13] S. Shamai and A.D. Wyner. Information theoretic considerations for symmetric, cellular, multiple-access fading channels part I. IEEE Transactions on Information Theory, 43(6):1877-1894, 1997.

[14] M. Médard, J. Huang, A. Goldsmith, S. Meyn, and T. Coleman. Capacity of time-slotted ALOHA packetized multiple-access systems over the AWGN channel. IEEE Transactions on Wireless Communications, 3(2):486-499, 2004.

[15] L. Georgiadis, M. Neely, and L. Tassiulas. Resource allocation and cross-layer control in wireless networks. Foundations and Trends in Networking, 1(1):1-144, 1006.

[16] T. Cover, R. McEliece, and E. Posner. Asynchronous multiple-access channel capacity. IEEE Transactions on Information Theory, 27(4):409413, 1981.

[17] A. Parandehgheibi, A. Ozdaglar, M. Médard, and A. Eryilmaz. Dynamic rate allocation in fading multiple access channels. In Proceedings of ITA, 2008. 